〈Research Paper〉

\title{
Effect of Ethanol/water Solvent Ratios on the Morphology of Zein Nanofiber Mats and their Wettability
}

\author{
Jin Hyun Choi, Young Hwa Kim and Jeong Hyun Yeum ${ }^{\dagger}$ \\ Department of Advanced Organic Materials Science and Engineering, Kyungpook National University, Daegu 702-701, Korea
}

(Received: June 1, 2011/Revised: June 10, 2011/Accepted: September 26, 2011)

\begin{abstract}
Zein is a hydrophobic protein produced from maize and has great potential in a number of industrial applications, such as food, food coating and food packaging. To obtain suitable electrospinning conditions for thinner and uniform zein nanofiber mats, a series of experiments was conducted on various volume ratios $(\mathrm{v} / \mathrm{v})$ of ethanol/water solutions with different zein concentrations. The prepared zein nanofiber mats were characterized by field emission scanning electron microscopy and contact angle measurements. Uniform zein fibers with a average diameter in the nanometer scale $(300 \sim 500 \mathrm{~nm})$ could be prepared from 30 wt. $\%$ zein in $7 / 3(\mathrm{v} / \mathrm{v})$ ethanol/water solutions.
\end{abstract}

Keywords: electrospinning, nanofiber mats, zein, solvent ratio, wettability

\section{Introduction}

Electrospinning is a unique and convenient method for producing nanoscale fibers from both synthetic and natural polymers for a range of applications in biomedical engineering, filtration, protective clothing, catalysis reaction and sensors ${ }^{1,2)}$. The principle of the electrospinning method is quite simple. A high voltage is applied to create electrically charged jets of polymer solutions, which form nanofibers.

These nanofibers are collected on a collector as a nonwoven mat. Recently, a broad range of synthetic polymers and natural biopolymers have been electrospun discretely or mutually for the fabrication of nanofiber mats for a variety of applications ${ }^{3-14)}$.

Zein is the major storage protein of corn and accounts for $40-50 \%$ of its protein content; it is soluble in aqueous alcohol solutions (60-95\%). This agro-based natural polymer has more hydrophobic characteristics than other proteins due to the presence of the apolar amino acids, proline and glutamine, which are the main constituents of zein. Because zein is one of most hydrophobic, low toxic, renewable and biodegradable proteins, it has been used widely in the packaging, food, pharmaceutical, cosmetic and biomedical industries ${ }^{15-20)}$.
In this study, zein nanofiber mats were prepared by electrospinning, using aqueous ethanol solutions as a solvent.

The effects of the ethanol/water ratios on the morphology of the zein nanofiber mats and their wettability were examined by field-emission scanning electron microscopy (FE-SEM) and contact angle measurements.

\section{Experimental}

\subsection{Materials}

Zein extracted from corn (molecular weight $=35,000$ ) was obtained from Tokyo Chemical Industry Co. Ltd., Japan and used as received.

Zein could be dispersed readily in 50-95\% ethanol /water mixtures as well as in aqueous glacial acetic/alcohol solutions but not in either absolute alcohol or water.

In the present study, the Zein sample used contained mainly $\alpha$-Zein and was soluble in aqueous $70-90 \%$ ethanol solutions at room temperature.

Ethanol $(96 \%$ purity, Daejung Chemical \& Materials Co. Ltd., Korea) and doubly distilled water were used as the solvent to prepare all solutions.

${ }^{\dagger}$ Corresponding author. Tel.: +82-53-950-5739; Fax.: +82-53-950-6744; e-mail: jhyeum@knu.ac.kr

(C)2011 The Korean Society of Dyers and Finishers 1229-0033/2011-12/227-232 


\subsection{Electrospinning zein nanofiber mats}

To prepare the electrospinning solution, zein were dissolved at different volume ratios of ethanol/water of $9 / 1,8 / 2$ and $7 / 3(\mathrm{v} / \mathrm{v})$. The zein concentrations used were 20,25 and $30 \%$ based on the weight of the solution. During electrospinning, a high voltage power supply (CHUNGPA EMT Co., Korea) was applied to the electrospinning solution contained in a syringe via an alligator clip attached to the syringe needle. The applied voltage was adjusted to $10 \mathrm{kV}$. The solution was delivered to the blunt needle tip via a syringe pump to control the solution flow rate. The fibers were collected on an electrically grounded aluminum foil placed at a $15 \mathrm{~cm}$ vertical distance to the needle tip. Fig. 1 shows a schematic representation of the electrospinning process.

\subsection{Characterizations}

The morphology of zein nanofiber mats was observed with a FE-SEM (JEOL, model JSM-6380) after the gold coating. The surface wettability of the nanofiber mats was evaluated by contact angle measurements using a DSA100 contact angle meter

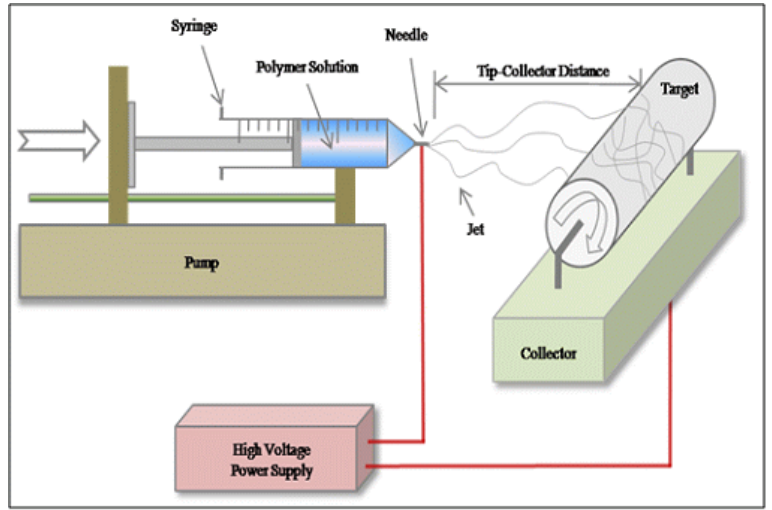

Fig. 1. Schematic diagram of electrospinning process.

(Kruss, Germany). The images were recorded on a CCD camera immediately after the water drop had been deposited onto the nanofiber mat surface, and the mean contact angles were determined using Kruss image analysis software. The contact angles were measured at $25^{\circ} \mathrm{C}$ and at least ten measurements per mat were performed.

\section{Results and Discussion}

Figs. 2, 3 and 4 show the effect of the polymer concentration and volume ratios ( $\mathrm{v} / \mathrm{v})$ of ethanol/ water on the nanofiber diameter and morphology.
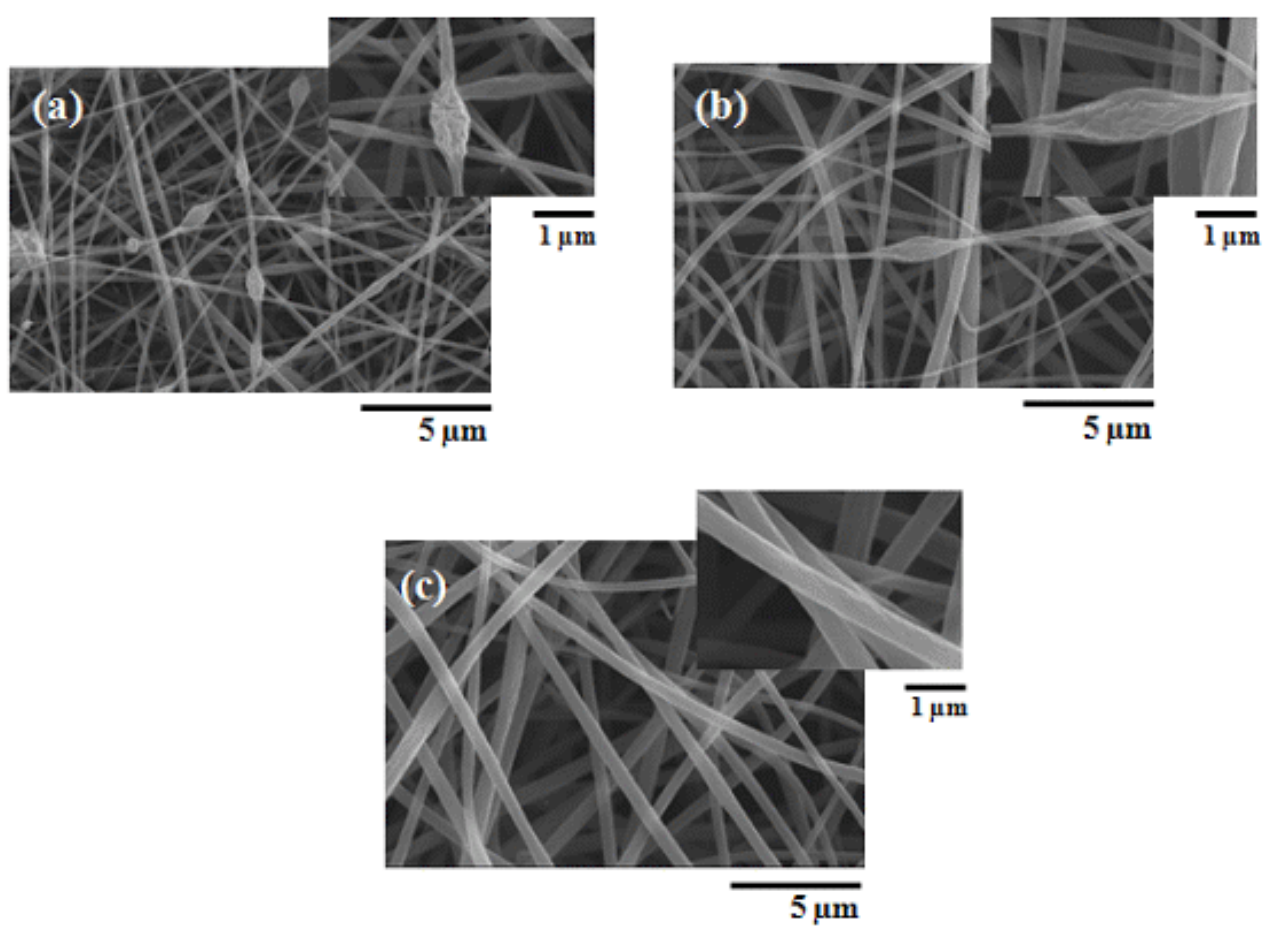

Fig. 2. FE-SEM images of zein nanofiber mats electrospun from aqueous ethanol solutions with an ethanol/water ratio of $7 / 3(\mathrm{v} / \mathrm{v})$ at different zein concentrations of (a) 20 wt.\%, (b) 25 wt.\% and (c) 30 wt.\% (inset: high magnification images). 
To obtain suitable electrospinning conditions for thinner and uniform zein nanofiber mats, a series of experiments were conducted on various volume ratios $(\mathrm{v} / \mathrm{v})$ of ethanol/water solutions and different zein concentrations. Fig. 2 shows the typical morphology of the 20,25 and 30 wt.\% of zein solution concentration in $7 / 3(\mathrm{v} / \mathrm{v})$ of ethanol/water solutions at a fixed applied voltage $(10 \mathrm{kV})$ and the tip to collector distance $(15 \mathrm{~cm})$. At low polymer solution concentrations (20 and 25 wt.\%), fibers connected by some beads were found, as shown in Fig. 2a and $2 b$. As the zein concentration was increased, the bead density decreased gradually.

When the zein concentration was 30 wt.\% (Fig. 2c), uniform zein nanofiber mats with no bead defects were observed. A nanometer range of ultrafine electrospun nanofiber mats $(300 \sim 500 \mathrm{~nm})$ were obtained in the aqueous solutions, as shown in Fig. 2c. As we know these formation of beads and beaded fibers is driven by the surface tension.

Surface tension tries to make the surface area per unit mass smaller, by changing the jets into spheres, the forces from the excess charge try to increase the surface area, which opposes the formation of beads and favors thinner jets; viscoelastic force resists rapid changes in shape. The major competition is between the surface tension and viscoelastic force. Increasing the viscosity favors the formation of smooth fibers ${ }^{21)}$.

To evaluate the effect of the different volume ratios of ethanol/water in the polymer solution, the ethanol content was varied from 70 to 90 vol.\% and the results are shown in Fig. 3 and 4.

The prepared zein nanofiber mats showed a similar trend of morphological changes. Some beads were found at low polymer solution concentrations but the prepared nanofibers showed a ribbon shape with a larger diameter (Fig. 5). The appearance of nanofibers was estimated to be related to the selfassembly of the protein during ethanol evaporation. The rapid evaporation of the solvent resulted in circular fibers that were elliptical and then flat, forming a ribbon-like structure.

The water contact angle data, which are indicatives of the wettability of mat surfaces, were obtained at $25^{\circ} \mathrm{C}$. As mentioned before, the zein proteins have more hydrophobic characteristics than other proteins as a consequence of the presence of the apolar amino
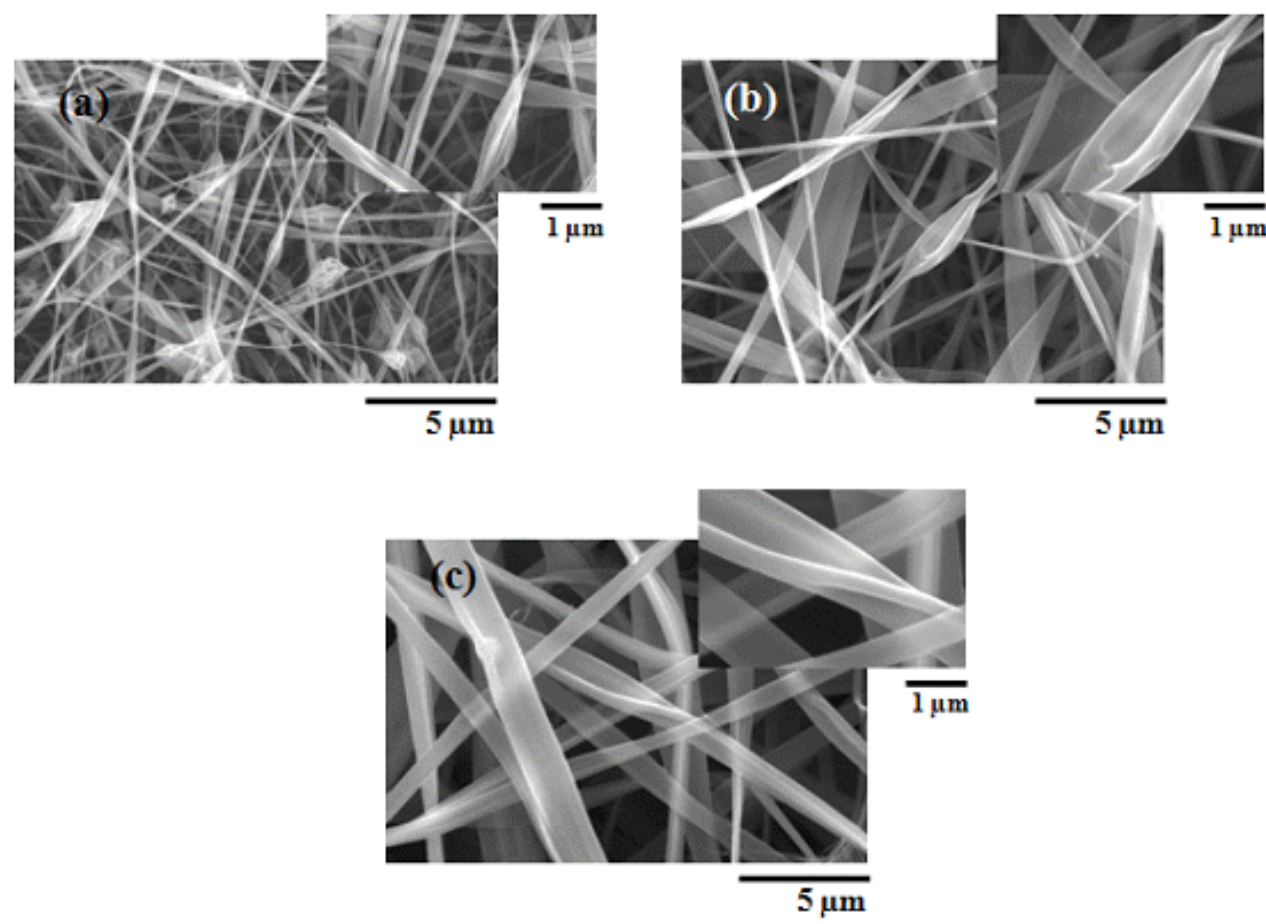

Fig. 3. FE-SEM images of zein nanofiber mats electrospun from aqueous ethanol solutions with an ethanol/water ratio of $8 / 2(\mathrm{v} / \mathrm{v})$ at different zein concentrations of (a) $20 \mathrm{wt} . \%$, (b) $25 \mathrm{wt} . \%$ and (c) 30 wt.\% (inset: high magnification images). 

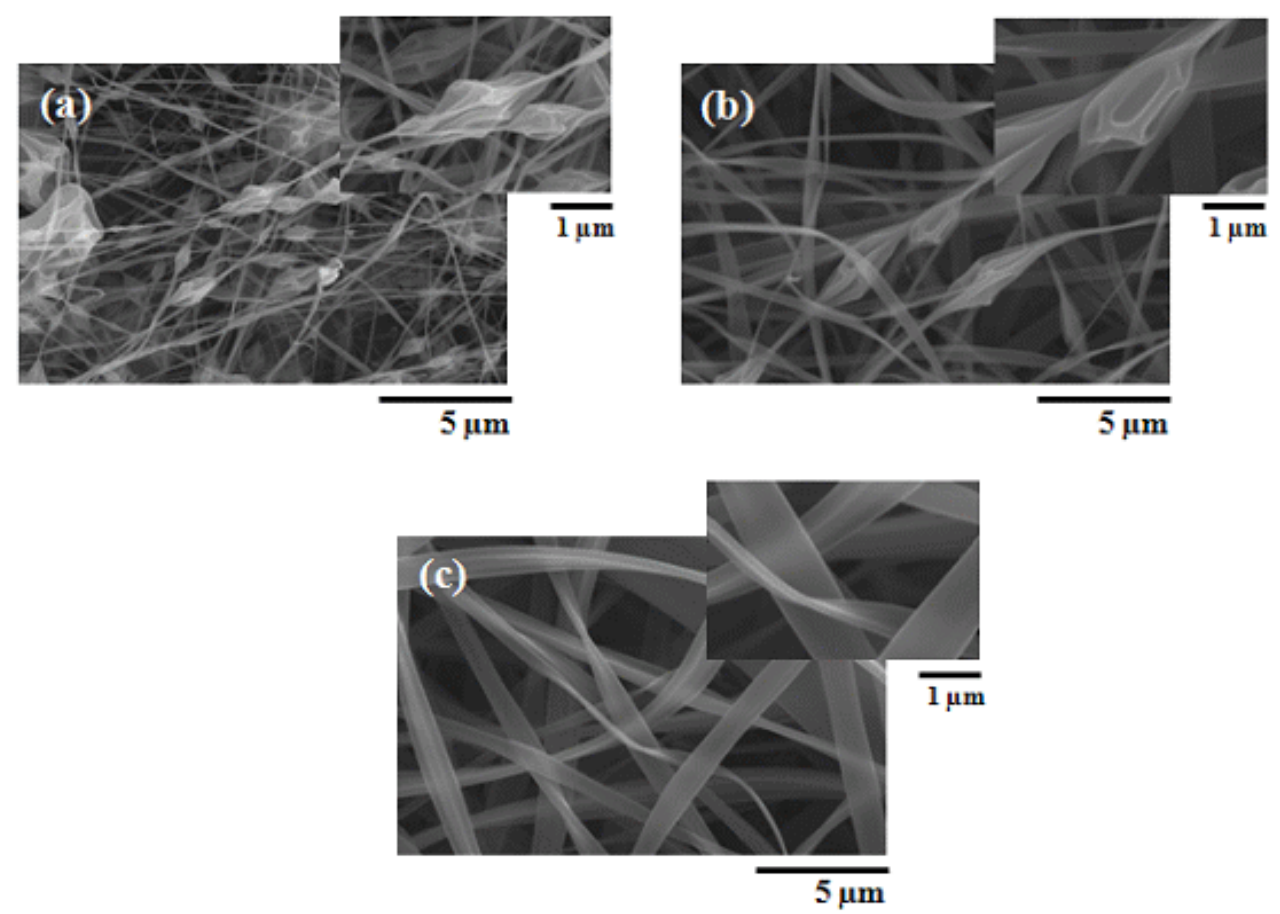

Fig. 4. FE-SEM images of zein nanofiber mats electrospun from aqueous ethanol solutions with an ethanol/water ratio of $9 / 1(\mathrm{v} / \mathrm{v})$ at different zein concentrations of (a) $20 \mathrm{wt} . \%$, (b) $25 \mathrm{wt} . \%$ and (c) 30 wt.\% (inset: high magnification images).

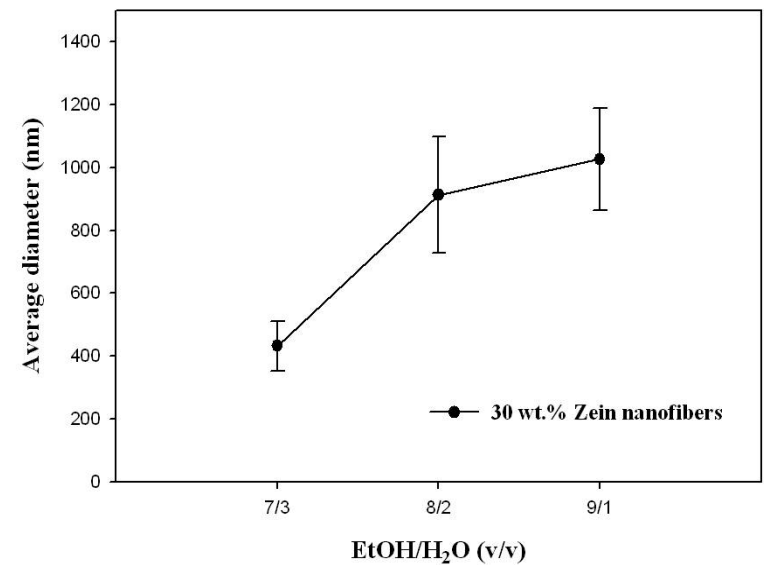

Fig. 5. Average diameter of zein nanofiber mats with different ethanol/water ratios.

acids proline and glutamin, which are the main constituents of zein. In this study, hydrophobicity of zein was analyzed in terms of structure ${ }^{20)}$.

A pristine zein film and zein nanofiber mats were characterized through contact angle measurements to determine the hydrophilicity of each nanofiber mat. The water contact angle will increase with increasing surface hydrophobicity. According to Fig. 6, the contact angle of the electrospun zein nanofiber mats was much higher than the zein film, which was made by a casting method.

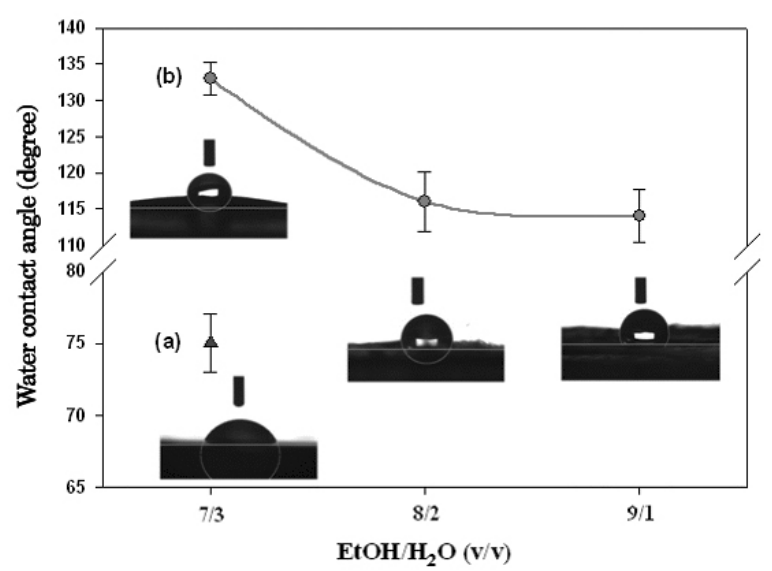

Fig. 6. Contact angle of (a) zein film and (b) electrospun zein nanofiber mats with different ethanol/water ratios.

Ultrafine zein nanofiber mats have a mean initial contact angle of approximately $133^{\circ}$, which is approximately twice as large as that of the zein film $\left(74^{\circ}\right)$. The nanofibrous network structure is believed to help enhance the hydrophobicity because the shape and surface roughness are very important factors in wetting ${ }^{21-25)}$.

\section{Conclusions}

The morphology of the electrospun nanofibers can be affected by different volume ratios ( $\mathrm{v} / \mathrm{v})$ of ethanol/ 
water solutions and zein concentration. The solvent mixture plays important roles on the electrospinnability of the bulk zein solutions as well as the morphology of the electrospun nanofiber mats.

Uniform zein nanofiber mats with a average diameter in the nanometer-scale $(300-500 \mathrm{~nm})$ could be prepared from the $7 / 3(\mathrm{v} / \mathrm{v})$ of ethanol/water solution with 30 wt.\% of zein. The wettability of the zein nanofiber mat depends on its shape, which showed high hydrophobicity.

\section{Acknowledgements}

This research was financially supported by the Ministry of Education Science Technology (MEST) and Korea Institute for Advancement of Technology (KIAT) through the Human Resource Training Project for Regional Innovation. Also, this work was partially supported in part by grants from the Agenda Program (PJ0073852010) of National Institute of Crop Science, Rural Development Administration (RDA), Korea.

\section{References}

1. D. H. Reneker and I. Chun, Nanometre Diameter Fibres of Polymer, Produced by Electrospinning, Nanotechnology, 7(3), 216-223(1996).

2. D. Li and Y. Xia, Electrospinning of Nanofibers: Reinventing the Wheel?, Adv. Mater., 16(14), 1151-1170(2004).

3. L. Zhao, C. He, Y. Gao, L. Cen, L. Cui, and Y. Cao, Preparation and Cytocompatibility of PLGA Scaffolds with Controllable Fiber Morphology and Diameter Using Electrospinning Method, $J$. Biomed. Mater. Res. B, 87(1), 26-34(2008).

4. J. S. Choi, S. J. Lee, G. J. Christ, A. Atala, and J. J. Yoo, The Influence of Electrospun Aligned Poly(3-caprolactone)/Collagen Nanofiber Meshes on the Formation of Self-Aligned Skeletal Muscle Myotubes, Biomaterials, 29(19), 2899-2906(2008).

5. E. J. Chong, T. T. Phan, I. J. Lim, Y. Z. Zhang, B. H. Bay, S. Ramakrishna, and C. T. Lim, Evaluation of Electrospun PCL/Gelatin Nanofibrous Scaffold for Wound Healing and Layered Dermal Reconstitution, Acta Biomater., 3(3), 321-330(2007).
6. M. Li, M. J. Mondrinos, X. Chen, M. R. Gandhi, F. K. Ko, and P. I. Lelkes, CoElectrospun Poly(lactide-co-glycolide), Gelatin, and Elastin Blends for Tissue Engineering Scaffolds, J. Biomed. Mater. Res. A, 79(4), 963973(2006).

7. H. W. Lee, M. R. Karim, J. H. Park, H. D. Ghim, J. H. Choi, K. Kim, Y. Deng, and J. H. Yeum, Poly(vinyl alcohol)/Chitosan Oligosaccharide Blend Submicrometer Fibers Prepared from Aqueous Solutions by the Electrospinning Method, J. Appl. Polym. Sci., 111(1), 132-140(2009).

8. H. W. Lee, M. R. Karim, J. H. Park, D. G. Bae, W. Oh, I. W. Cheong, and J. H. Yeum, Electrospinning and Characterisation of Poly(vinyl alcohol) Blend Submicron Fibers in Aqueous Solution, Polym. Compos., 17(1), 47-54(2009).

9. H. W. Lee, M. R. Karim, H. M. Ji, J. H. Choi, H. D. Ghim, S. M. Park, W. Oh, and J. H. Yeum, Electrospinning Fabrication and Characterization of Poly(vinyl alcohol)/Montmorillonite Nanofiber Mats, J. Appl. Polym. Sci., 113(3), 1860-1867 (2009).

10. M. R. Karim, H. W. Lee, R. Kim, B. C. Ji, J. W. Cho, T. W. Son, W. Oh, and J. H. Yeum, Preparation and Characterization of Electrospun Pullulan/Montmorillonite Nanofiber Mats in Aqueous Solution, Carbohyd. Polym., 78(2), 336342(2009).

11. J. H. Park, H. W. Lee, D. K. Chae, W. Oh, J. D. Yun, Y. Deng, and J. H. Yeum, Electrospinning and Characterization of Poly(vinyl alcohol)/ Chitosan Oligosaccharide/Clay Nano-composite Nanofibers in Aqueous Solution, Colloid Polym. Sci., 287(8), 943-950(2009).

12. H. M. Ji, H. W. Lee, M. R. Karim, I. W. Cheong, E. A. Bae, T. H. Kim, M. S. Islam, B. C. Ji, and J. H. Yeum, Electrospinning and Characterization of Medium-Molecular-Weight Poly(vinyl alcohol)/High-Molecular-Weight Poly (vinyl alcohol)/Montmorillonite Nanofibers, Colloid Polym. Sci., 287(7), 751-758(2009).

13. J. H. Park, M. R. Karim, I. K. Kim, I. W Cheong, J. W. Kim, J. W. Cho, and J. H. Yeum, Electrospinning Fabrication and Characterization 
of Poly(vinyl alcohol)/Montmorillonite/Silver Hybrid Nanofibers for Antibacterial Applications, Colloid Polym. Sci., 288(1), 115-121(2010).

14. J. H. Yang, N. S. Yoon, J. H. Park, I. K. Kim, I. W. Cheong, Y. Deng, W. Oh, and J. H. Yeum, Electrospinning Fabrication and Characterization of Poly(vinyl alcohol)/Waterborne Polyurethane Nanofiber Membranes in Aqueous Solution, $J$. Appl. Polym. Sci., 120(4), 2337-2345(2011).

15. R. Shukla and M. Cheryan, Zein: the Industrial Protein from Corn, Ind. Crop. Prod., 13(3), 171192(2001).

16. T. Miyoshi, K. Toyohara, and H. Minematsu, Preparation of Ultrafine Fibrous Zein Membranes via Electrospinning, Polym. Int., 54(8), 11871190(2005).

17. S. Torres-Giner, E. Gimenez, and J. M. Lagaron, Characterization of the Morphology and Thermal Properties of Zein Prolamine Nanostructures Obtained by Electrospinning, Food Hydrocolloid., 22(4), 601-614(2008).

18. C. Yao, X. Li, and T. Song, Electrospinning and Crosslinking of Zein Nanofiber Mats, $J$. Appl. Polym. Sci., 103(1), 380-385(2007).

19. C. Yao, X. Li, and T. Song, Fabrication of Zein/Hyaluronic Acid Fibrous Membranes by
Electrospinning, J. Biomat. Sci-polym. E., 18(6), 731-742(2007).

20. S. Torres-Giner, M. J. Ocio, and J. M. Lagaron, Novel Antimicrobial Ultrathin Structures of Zein/ Chitosan Blends Obtained by Electrospinning, Carbohyd. Polym., 77(2), 261-266(2009).

21. H. Fong, I. Chun and D. H. Reneker, Beaded Nanofibers Formed during Electospinning, Polymer, 40(16), 4585-4592(1999).

22. L. Jiang, R. Wang, B. Yang, T. J. Li, D. A. Trigk, A. Fujishima, K. Hashimoto, and D. Zhu, Binary Cooperative Complementary Nanoscale Interfacial Materials, Pure Appl. Chem., 72(1-2), 73-81(2000).

23. L. Feng, S. Li, Y. Li, H. Li, L. Zhang, J. Zhai, Y. Song, B. Liu, L. Jiang, and D. Zhu, Super-Hydrophobic Surfaces: from Natural to Artificial, Adv. Mater., 14(24), 1857-1860(2002).

24. K. Acatay, E. Simsek, C. Ow-Yang, and Y. Z. Menceloglu, Tunable Superhydrophobically Stable Polymeric Surfaces by Electrospinning, Angew. Chem. Int. Ed., 43(39), 5210-5213(2004).

25. S. M. Park, I. J. Kwon, J. Y. Kim, C. N. Kim, J. H. Yeum, and N. S. Yoon, A Study on the Super-hydrophobicity of Poly(ethylene terephthalate) Fabric by $\mathrm{TiO}_{2}$ Nanoparticles Coating, Textile Coloration and Finishing, 21(1), 30-37(2009). 